CHAPTER 9.

Cosmology 


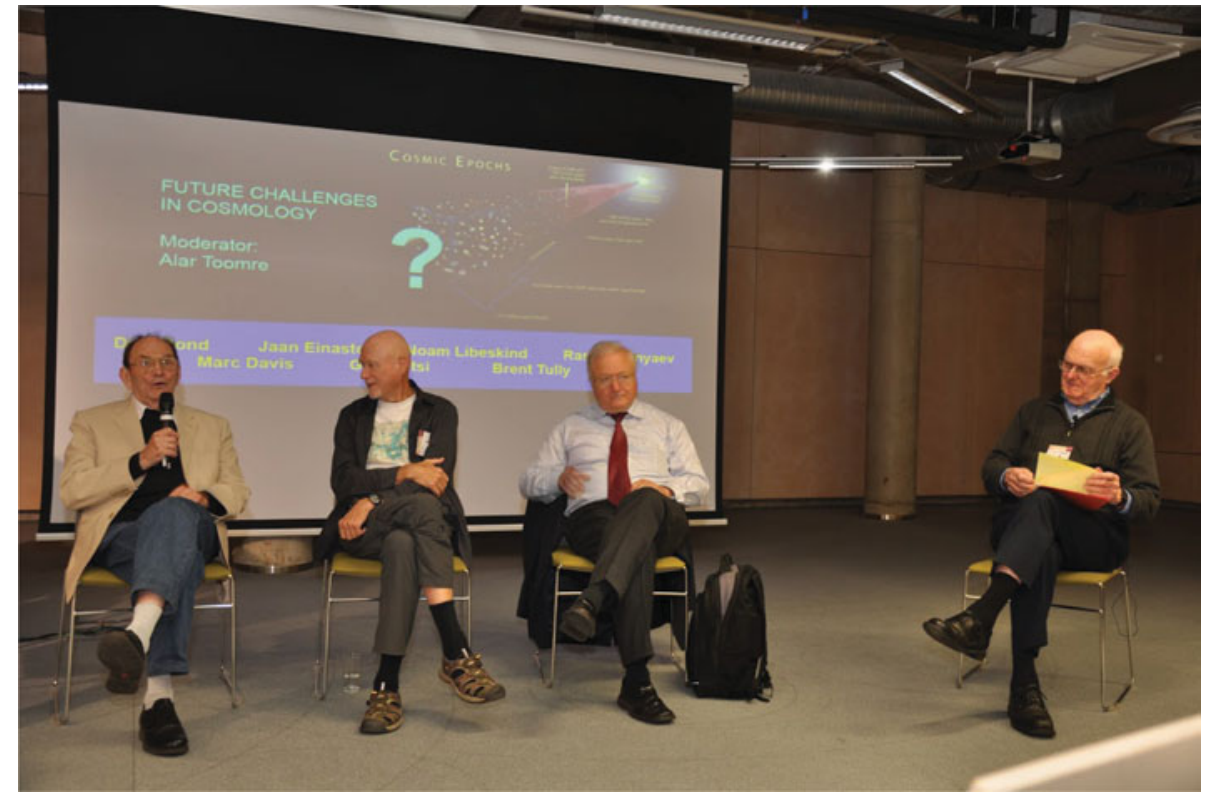

Gruber Prize cosmology panel in action:

Moderator Alar Toomre in discussion with

Jaan Einasto, Brent Tully and Rashid Sunyaev

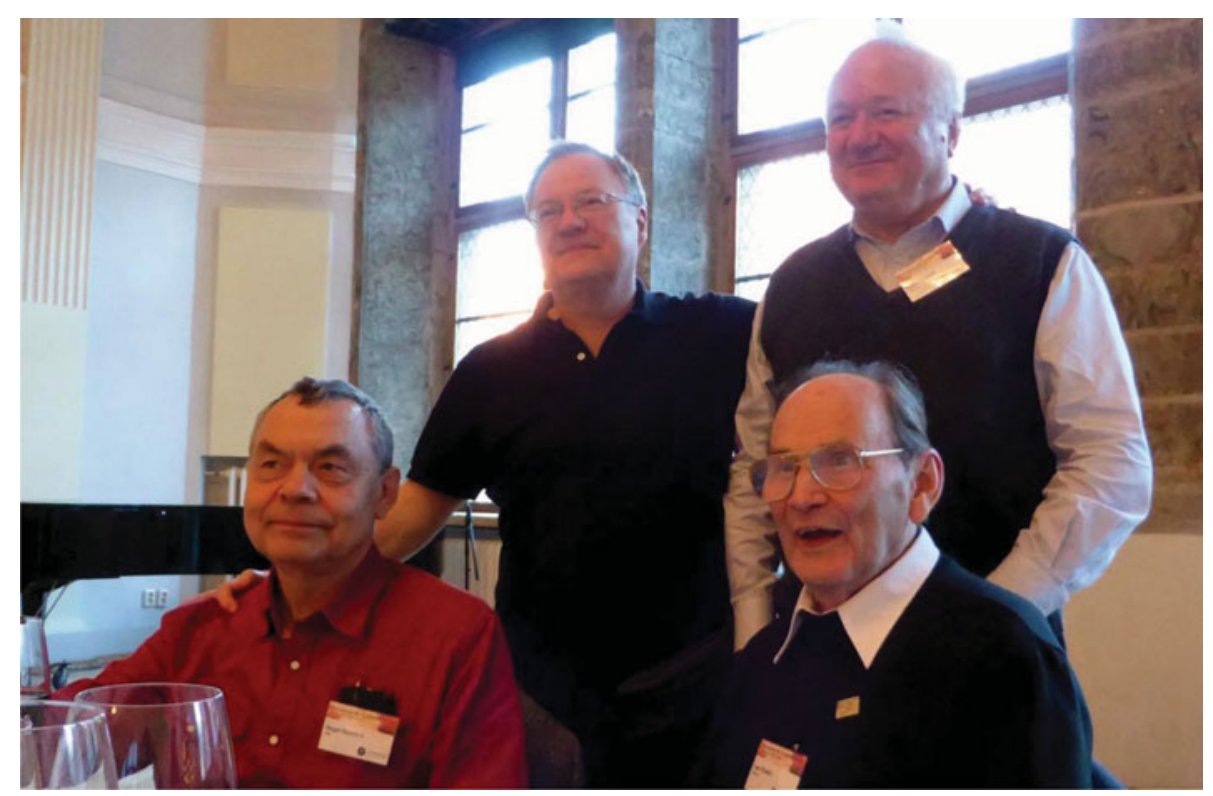

Cosmology in high spirits:

Sergei Shandarin \& Jaan Einasto (sitting),

Dick Bond \& Rashid Sunyaev (standing) 


\title{
Probing the accelerating Universe with redshift-space distortions in VIPERS
}

\author{
Sylvain de la Torre ${ }^{1}$ and VIPERS collaboration \\ ${ }^{1}$ Aix Marseille Université, CNRS, LAM (Laboratoire d'Astrophysique de Marseille) UMR 7326, \\ 13388, Marseille, France \\ email: sylvain.delatorre@lam.fr
}

\begin{abstract}
We present the first measurement of the growth rate of structure at $z=0.8$. It has been obtained from the redshift-space distortions observed in the galaxy clustering pattern in the VIMOS Public Redshift survey (VIPERS) first data release. VIPERS is a large galaxy redshift survey probing the large-scale structure at $0.5<z<1.2$ with an unprecedented accuracy. This measurement represents a new reference in the distant Universe, which has been poorly explored until now. We obtain $f \sigma_{8}=0.47 \pm 0.08$ at $z=0.8$ that is consistent with the predictions of standard cosmological models based on Einstein gravity. This measurement alone is however not accurate enough to allow the detection of possible deviations from standard gravity.
\end{abstract}

Keywords. large-scale structure of universe, cosmological parameters, cosmology: observations

\section{Introduction}

The cosmological model is today well established with regards to our knowledge of the amount of Universe's major constituents (e.g. Planck Collaboration, 2013). This has been achieved thanks to geometrical probes such as cosmic microwave background anisotropies (CMB), baryonic acoustic oscillations in the galaxy clustering (BAO) or type 1a supernovae distance-redshift measurements (SN1a). The later has permitted to evidence that the Universe was experiencing a late-time accelerated expansion due to an unknown dark energy component dominating the total energy density today (Riess et al., 1998; Perlmutter et al., 1999). However, measurements of the expansion history of the Universe from geometrical probes alone, cannot discriminate dark energy from modifications to standard gravity to explain the observed acceleration of expansion. This degeneracy can only be lifted by measuring the growth rate of structure at different epochs $f(z)$ (e.g. Peacock et al., 2006).

Galaxy redshift surveys provide a wealth of cosmological information, mapping the late-time development of the small metric fluctuations that existed at early times, and whose early properties can be viewed in the CMB. The growth of structure during this intervening period is sensitive both to the type and amount of dark matter, and also to the theory of gravity, so there is a strong motivation to make precise measurements of the rate of growth of structure. The main advantage of redshift surveys is that the radial information depends on the expansion history and is corrupted by peculiar velocities. The peculiar velocities induce an anisotropy in the apparent clustering, the redshiftspace distortions, from which the properties of peculiar velocities can be inferred much more precisely than in any attempt to measure them directly using distance estimators. Because these peculiar velocities on large scales are induced by structure growth, redshiftspace distortions is a unique probe of the growth rate of structure.

The possibility of using the redshift-space distortion signature as a probe of the growth rate of structure, together with that of using $\mathrm{BAO}$ as a standard ruler to measure the 
expansion history, is one of the main reasons behind the recent burst of activity in galaxy redshift surveys. The first paper to emphasise this application as a test of gravity theories was the analysis of the VVDS survey by Guzzo et al. (2008) and subsequent work using larger surveys has exploited this method to make measurements at $z<1$ (e.g. Cabré \& Gaztañaga, 2009; Beutler et al., 2012). The prime goal of the VIMOS Public Redshift survey (VIPERS) is to provide an accurate measurement of the growth rate of structure at redshift around unity (Guzzo et al., 2014). We report here the initial redshift-space distortions analysis in VIPERS, performed in de la Torre et al. (2013).

\section{Redshift-space distortions in VIPERS}

The VIPERS survey is intended to provide robust and precise measurements of the properties of the galaxy population at an epoch when the Universe was about half its current age. It covers $24 \mathrm{deg}^{2}$ on the sky, divided over two areas within the W1 and W4 CFHTLS fields. Galaxies are selected to a limit of $i_{A B}<22.5$ and by applying a simple and robust gri colour preselection to efficiently remove galaxies at $z<0.5$. VIPERS spectra has been collected with the VIMOS multi-object spectrograph at moderate resolution $(\mathrm{R}=210)$. VIPERS observations are on-going and the dataset used in this analysis corresponds to the VIPERS Public Data Release 1 (PDR-1) catalogue. This catalogue includes 55358 redshifts and represents $64 \%$ of the final survey in terms of covered area. The catalogue that we use to perform the redshift-space distortions analysis corresponds to a sub-sample of 45871 galaxies with reliable redshift measurements. A complete description of the survey and the PDR-1 catalogue are given in Guzzo et al. (2014) and Garilli et al. (2014).

The galaxy clustering in the VIPERS sample is characterized by measuring the twopoint statistics of the spatial distribution of galaxies in configuration space, using the two-point correlation function. VIPERS has a complex angular selection function which has to be taken into account carefully when estimating the correlation function. For this, a detailed correction scheme has been developed that is described in de la Torre et al. (2013). In order to measure the anisotropy induced by structure growth, we measure the multipole moments of the anisotropic correlation function. We effectively measure the two first non-null moments $\xi_{0}(s)$ and $\xi_{2}(s)$, where most of the relevant information is contained.

The measured $\xi_{0}(s)$ and $\xi_{2}(s)$ for all galaxies between $0.7<z<1.2\left(z_{\text {med }}=0.8\right)$ in the VIPERS sample are presented in Fig. 1. To derive the growth rate of structure, we fit the Scoccimarro (2004) model to the measurements, defining the galaxy bias parameter times the normalisation of the matter power spectrum $b \sigma_{8}$, the pairwise velocity dispersion $\sigma_{v}$, and the growth rate of structure times the normalisation of the matter power spectrum $f \sigma_{8}$, as free parameters. We assume a fixed shape of the mass power spectrum consistent with the cosmological parameters obtained from WMAP9 (Hinshaw et al., 2013) and perform a maximum likelihood analysis. The best-fitting models are shown in Fig. 1 when considering either a Gaussian or a Lorentzian damping function to reproduce the smallscale distortions induced by the Fingers-of-God effect (see de la Torre \& Guzzo, 2012). After marginalising over $\sigma_{v}$ and $b \sigma_{8}$ parameters, we obtain a value of $f \sigma_{8}=0.47 \pm 0.08$.

Our measurement of $f \sigma_{8}$ is shown in Fig. 2 and compared with previous measurements and predictions for various cosmological models. This measurement is consistent with the General Relativity prediction in a flat $\Lambda$ CDM Universe with cosmological parameters given by WMAP9 and Planck experiments, for which the expected values are $f(0.8) \sigma_{8}(0.8)=0.45$ and $f(0.8) \sigma_{8}(0.8)=0.46$ respectively. We find that our result is not significantly altered if we adopt a Planck cosmology for the shape of the 


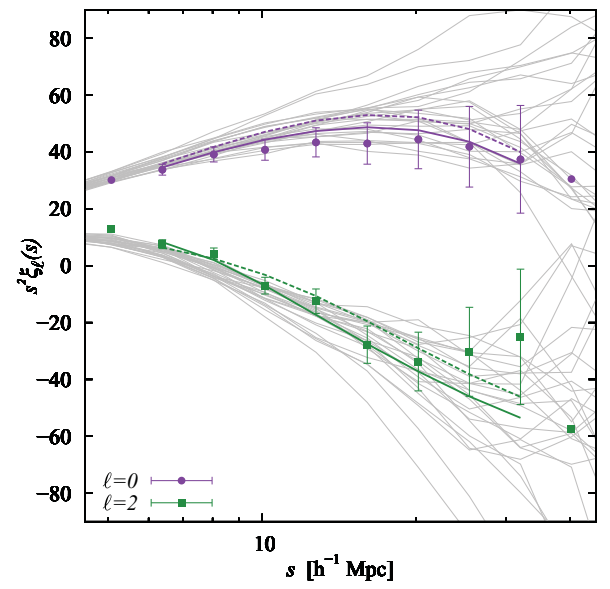

Figure 1. The monopole and quadrupole moments of the redshift-space correlation function, $\xi_{0}(s)$ and $\xi_{2}(s)$, as measured in the VIPERS sample. The shallow curves show the results for the 26 mock survey realisations. The points are for the measured VIPERS data at $0.7<z<1.2$. The solid and dotted lines correspond to the best-fitting models to the data with different damping functions describing small-scale distortions. Figure reproduced from de la Torre et al. (2013).

mass power spectrum. It is also in good agreement with previous measurements at lower redshifts from 2dFGRS (Hawkins et al., 2003), 2SLAQ (Ross et al., 2007), VVDS (Guzzo et al., 2008), SDSS LRG (Cabré \& Gaztañaga, 2009; Samushia et al., 2012), WiggleZ (Blake et al., 2012), BOSS (Reid et al., 2012), and 6dFGS (Beutler et al., 2012) surveys. In particular, it is compatible within $1 \%$ with the results obtained in the VVDS and WiggleZ surveys at a similar redshift, although WiggleZ measurements tend to suggest lower $f \sigma_{8}$ values, smaller than expected in standard gravity (but see Contreras et al., 2013). Finally we compare our measurement to the predictions of three of the most plausible modified gravity models, studied in di Porto et al. (2012). We consider Dvali-Gabadadze-Porrati (Dvali et al., 2000), $f(R)$, and coupled dark energy models and show their predictions in Fig. 2. We find that our $f \sigma_{8}$ measurement is currently unable to discriminate between these modified gravity models and standard gravity given the size of the uncertainty, although we expect to improve the constraints with the analysis of the VIPERS final dataset.

\section{Conclusions}

We have analysed the redshift-space clustering properties of galaxies in the VIPERS survey first data release. The first data release of about 54000 galaxies at $0.5<z<1.2$ in the VIPERS survey allows a measurement of the redshift-space clustering of galaxies to an unprecedented accuracy over the redshift range $0.5<z<1.2$. The main goal of VIPERS is to provide an accurate measurement of the growth rate of structure through the characterisation of the redshift-space distortions in the galaxy clustering pattern. With the first data release we have been able to provide an initial measurement of $f \sigma_{8}$ at $z=0.8$. We find a value of $f \sigma_{8}=0.47 \pm 0.08$ which is in agreement with previous measurements at lower redshifts. This allows us to put a new constraint on gravity at the epoch when the Universe was almost half its present age. Our measurement of $f \sigma_{8}$ is statistically consistent with a Universe where the gravitational interactions between structures on $10 h^{-1}$ Mpc scales can be described by Einstein's theory of gravity. 


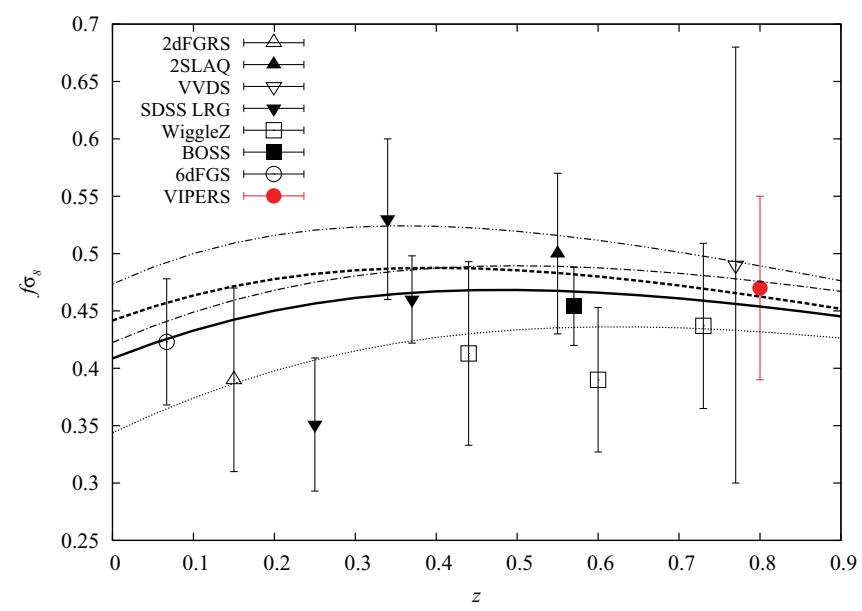

Figure 2. VIPERS $f \sigma_{8}$ initial measurement compared with a compilation of recent results from various surveys (see inset). The thick solid (dashed) curve corresponds to the prediction for General Relativity in a $\Lambda$ CDM model with WMAP9 (Planck) parameters, while the dotted, dot-dashed, and dot-dot-dashed curves are respectively Dvali-Gabadaze-Porrati, coupled dark energy, and $f(R)$ model expectations. Figure reproduced from de la Torre et al. (2013).

The present dataset represents the half-way stage of the VIPERS project, and the final survey will be large enough to subdivide our measurements and follow the evolution of $f \sigma_{8}$ out to redshift one. This will allow us to address some issues such as the suggestion from the WiggleZ measurements that $f \sigma_{8}$ is lower than expected at $z>0.5$ Our measurement at $z=0.8$ already argues against such a trend to some extent, but the larger redshift baseline and tighter errors from the final VIPERS dataset can be expected to deliver a definitive verdict on the high-redshift evolution of the strength of gravity.

\section{References}

Blake, C., Brough, S., Colless, M., et al. 2012, MNRAS, 425, 405

Beutler, F., Blake, C., Colless, M., et al. 2012, MNRAS, 423, 3430

Cabré, A. \& Gaztañaga, E. 2009, MNRAS, 393, 1183

Contreras, C., Blake, C., Poole, G. B., et al. 2013, MNRAS, 430, 924

de la Torre, S. \& Guzzo, L. 2012, MNRAS, 427, 327

de la Torre, S., Guzzo, L., Peacock, J. A., et al. 2013, A\&A, 557, A54

di Porto, C., Amendola, L., \& Branchini, E. 2012, MNRAS, 419, 985

Dvali, G., Gabadadze, G., \& Porrati, M. 2000, Physics Letters B, 485, 208

Garilli, B., Guzzo, L., Scodeggio, M., et al. 2014, A\& A, 562, A23

Guzzo, L., Pierleoni, M., Meneux, B., et al. 2008, Nature, 451, 541

Guzzo, L., Scodeggio, M., Garilli, B., et al. 2014, A\&A, 566, A108

Hawkins, E., Maddox, S., Cole, S., et al. 2003, MNRAS, 346, 78

Hinshaw, G., Larson, D., Komatsu, E., et al. 2013, ApJS, 208, 19

Peacock, J. A., Schneider, P., Efstathiou, G., et al. 2006, ESA-ESO Working Group on Fundamental Cosmology, Edited by J. A. Peacock et al. ESA

Perlmutter, S., Aldering, G., Goldhaber, G., et al. 1999, ApJ, 517, 565

Planck Collaboration, Ade, P. A. R., Aghanim, N., et al. 2013, arXiv:1303.5076

Reid, B. A., Samushia, L., White, M., et al. 2012, MNRAS, 426, 2719

Riess, A. G., Filippenko, A. V., Challis, P., et al. 1998, AJ, 116, 1009

Ross, N. P., da Ângela, J., Shanks, T., et al. 2007, MNRAS, 381, 573

Samushia, L., Percival, W. J., \& Raccanelli, A. 2012, MNRAS, 420, 2102

Scoccimarro, R. 2004, PRD, 70, 083007 